\title{
Risk Factors for Cholecystectomy After Laparoscopic Roux-En-Y Gastric Bypass
}

\author{
Sylke Haal ${ }^{1,2}$ (1) Djoeke Rondagh ${ }^{1} \cdot$ Barbara A. Hutten $^{3} \cdot$ Yair I. Z. Acherman ${ }^{4}$ - Arnold W. J. M. van de Laar ${ }^{4}$. \\ Roeland Huijgen ${ }^{2} \cdot$ Victor E. A. Gerdes ${ }^{2,5} \cdot$ Rogier P. Voermans ${ }^{1}$
}

Published online: 19 November 2019

(C) The Author(s) 2019

\begin{abstract}
Background Patients who have undergone bariatric surgery are at risk for subsequent cholecystectomy. We aimed to identify risk factors for cholecystectomy after laparoscopic Roux-en-Y gastric bypass (LRYGB).

Methods We conducted a retrospective case-control study of patients who underwent LRYGB between 2013 and 2015. Cases underwent cholecystectomy because of biliary symptoms after LRYGB. For each case, two controls were selected without subsequent cholecystectomy. Logistic regression analyses were used to identify risk factors.

Results Between 2013 and 2015, 1780 primary LRYGBs were performed. We identified 233 (13.1\%) cases who had undergone cholecystectomy after a median (IQR) of 12 (8-17) months, and 466 controls. Female gender (OR (95\% CI) 1.83 (1.06-3.17)), Caucasian ethnicity (OR (95\% CI) $1.82(1.10-3.02)$ ), higher percent total weight loss (\%TWL) at 12 months (OR (95\% CI) 1.06 (1.04-1.09)), and preoperative pain syndrome (OR (95\% CI) $2.72(1.43-5.18))$ were significantly associated with an increased risk for cholecystectomy. Older age (OR (95\% CI) $0.98(0.96-0.99))$ and preoperative statin use were associated with a reduced risk (OR $(95 \% \mathrm{CI}) 0.56(0.31-1.00))$. A dose-effect relationship was found between the intensity of preoperative statin and risk for cholecystectomy.

Conclusions In our study, higher \% TWL and preoperative pain syndrome were associated with an increased risk for cholecystectomy besides the traditional risk factors female gender and Caucasian ethnicity. These factors can be used to identify high-risk patients, who might benefit from preventive measures. Whether statins can protect bariatric patients from developing gallstones should be investigated prospectively.
\end{abstract}

Keywords Cholecystectomy $\cdot$ Gallstones $\cdot$ Roux-en-Y gastric bypass $\cdot$ Risk factors

Electronic supplementary material The online version of this article (https://doi.org/10.1007/s11695-019-04166-y) contains supplementary material, which is available to authorized users.

Sylke Haal

s.haal@amsterdamumc.nl

Djoeke Rondagh

djoeke_r93@hotmail.com

Barbara A. Hutten

b.a.hutten@amsterdamumc.nl

Yair I. Z. Acherman

yacherman@spaarnegasthuis.nl

Arnold W. J. M. van de Laar

avandelaar@spaarnegasthuis.nl

Roeland Huijgen

rhuijgen@spaarnegasthuis.nl

Victor E. A. Gerdes

v.e.gerdes@amsterdamumc.nl
Rogier P. Voermans

r.p.voermans@amsterdamumc.nl

1 Department of Gastroenterology and Hepatology, Amsterdam UMC, Location AMC, Amsterdam Gastroenterology \& Metabolism, University of Amsterdam, Amsterdam, Netherlands

2 Department of Internal Medicine, Spaarne Gasthuis, Hoofddorp, Netherlands

3 Department of Clinical Epidemiology, Biostatistics and Bioinformatics, Amsterdam UMC, Location AMC, University of Amsterdam, Amsterdam, Netherlands

4 Department of Surgery, Spaarne Gasthuis, Hoofddorp, Netherlands

Department of Vascular Medicine, Amsterdam UMC, Location AMC, University of Amsterdam, Amsterdam, Netherlands 


\section{Introduction}

Patients with morbid obesity are at risk for symptomatic gallstone disease and cholecystectomy after bariatric surgery [1]. The incidence of gallstone formation as detected by ultrasonography after bariatric surgery can be up to 30 $45 \%$ [2, 3]. Overall, $8-15 \%$ of patients will develop symptoms within 2 years after surgery [4-7]. The majority of patients with gallstone disease suffer from biliary colic and are treated with laparoscopic cholecystectomy, which can be challenging due to adhesions caused by the previous bariatric surgery [8]. Furthermore, in case of choledocholithiasis, cholangitis, or biliary pancreatitis, conventional endoscopic retrograde cholangiopancreatography (ERCP) is not possible due to the altered anatomy and PTC or ERCP via gastrostomy is needed [9].

The increased risk on cholesterol gallstones after bariatric surgery involves several determinants including altered biliary cholesterol homeostasis during weight loss. Also gallbladder hypomobility, an increase in pro-nucleating factors, and an altered intestinal mobility seem to play a contributing role in the formation of cholesterol gallstones $[10,11]$.

In the general population, epidemiological studies have identified a number of risk factors, such as advanced age, female gender, Caucasian race, family history of gallstones, obesity-associated conditions, and rapid weight loss $[1,12]$. However, to our best knowledge, specific risk factors for cholecystectomy after bariatric surgery are not well established. Identification of risk factors in bariatric patients is important because it may provide opportunities for preventive therapy in high-risk patients. Until now, studies showed that weight loss, Caucasian race, female gender, advanced age, and insulin resistance are risk factors for (symptomatic) gallstones after bariatric surgery [2-5, 13-17]. Nevertheless, results of these studies were not consistent, studies were small in size, and majority of these studies focused on ultrasound-confirmed gallstones instead of symptomatic gallstones. We hypothesized that patients with a pain syndrome may have a higher risk for cholecystectomy given the higher prevalence of both gallstones and abdominal pains in patients after bariatric surgery.

Considering the steady increase of persons suffering from obesity and the recognized option to target obesity and its associated co-morbidity by means of bariatric interventions, the social and economic burden of gallstones post-bariatric surgery will further increase in the near future. Because of the heterogeneous results of previous performed studies, we aimed to identify risk factors for cholecystectomy after primary laparoscopic Roux-en-Y gastric bypass (LRYGB) in this retrospective study.

\section{Methods}

\section{Study Design and Patients}

We conducted a retrospective case-control study at the Medical Center (MC) Slotervaart (Amsterdam, the Netherlands). The local medical ethics committee confirmed that the Dutch Medical Research Involving Human Subjects Act did not apply to the current study and that an official approval was not required. All patients who underwent bariatric surgery in our institution have signed an informed consent form stating that their anonymized data can be used for scientific research. The electronic medical record system was searched to identify all patients (18-65 years) who underwent primary LRYGB between January 2013 and December 2015. Patients who underwent cholecystectomy because of postoperative biliary symptoms were defined as cases. Postoperative biliary symptoms consisted of biliary colic, cholecystitis, choledocholithiasis, cholangitis, or biliary pancreatitis (see "Study outcome" for the extensive definition of postoperative biliary symptoms). Only cases who developed biliary symptoms within 2 years after LYRGB were included, irrespective of the timing of the cholecystectomy. For each case, two patients who underwent LRYGB after that specific case and who remained free of biliary symptoms and cholecystectomy were consecutively selected as controls. The minimum period of follow-up was 24 months for all control patients. Exclusion criteria were prior or concomitant gallbladder surgery, prior bariatric surgery, another type of bariatric surgery than LRYGB, or the development of symptomatic gallstones more than 2 years after LRYGB. In our institute, an ultrasound is not regularly performed pre- or postoperatively and a concomitant cholecystectomy is only performed in case of symptomatic gallstone disease prior to bariatric surgery.

\section{Data Collection}

We collected preoperative data on demographics (age, gender, ethnicity, body mass index (BMI), physical activity; only for women: fertility, offspring, use of birth control pills), presence of comorbidity (hypertension, type 2 diabetes (T2D), and pain syndrome), and the preoperative use of medication. In addition, information on weight loss was gathered 12 months postoperative (window 6-18 months). In our outpatient clinic, weight loss is assessed during planned visits regularly at 6 , 12, and 24 months after LRYGB. Percent total weight loss was calculated by the following formula: \%TWL $=[$ (Initial Weight $)-($ Postoperative Weight)]/Initial Weight $\times 100$. Physical activity was determined according to the Dutch activity guide line for adults [18]. Hypertension was defined as known hypertension, use of antihypertensive medication, a systolic blood pressure $\geq 140 \mathrm{~mm} / \mathrm{Hg}$, or a diastolic blood pressure $\geq 90 \mathrm{~mm} / \mathrm{Hg}$; T2D as known T2D, use of 
antidiabetics, or $\mathrm{HbA} 1 \mathrm{c} \geq 53 \mathrm{mmol} / \mathrm{mol}$ at preoperative blood testing; fertile as age $\leq 50$ years or use of birth control pills; and preoperative pain syndrome as patients with a medical history of irritable bowel syndrome (IBS), fibromyalgia, or complex regional pain syndrome (CRPS). The individual potency of each statin was determined according to the metaanalysis by Law et al. [19, 20]. Four subgroups were defined for statin use: no statin use, low intensity (less than 1.6 times reduction in low-density lipoprotein cholesterol (LDL-C)), moderate intensity (1.6 times reduction, e.g., most widely prescribed dose of simvastatin $40 \mathrm{mg}$ daily), or high intensity (more than 1.6 times reduction in LDL-C).

\section{Study Outcome}

Primary outcome was the need for cholecystectomy because of postoperative biliary symptoms. Postoperative biliary symptoms consisted of biliary colic, cholecystitis, choledocholithiasis, cholangitis, or biliary pancreatitis. Biliary colic was defined as upper abdominal pain lasting at least $30 \mathrm{~min}$ with gallstones visible on abdominal imaging, according to the Rome criteria. Acute cholecystitis was defined according to the diagnostic criteria of the Tokyo Guidelines (TG18/TG13); there must be at least one local sign of inflammation: (1) Murphy's sign and (2) right upper quadrant mass/pain/tenderness, and at least one systemic sign of inflammation: (1) fever, (2) elevated C- reactive protein; (3) and elevated white blood cell count [21]. Choledocholithiasis was defined as the presence of stones in the intra- or extrahepatic bile ducts. Cholangitis was also defined according to the diagnostic criteria of the Tokyo Guidelines (TG18/TG13): signs of systematic inflammation, cholestasis, and evidence on imaging must be present [22]. Furthermore, acute biliary pancreatitis was diagnosed when the following features were present: (1) upper abdominal pain, (2) increased serum lipase or amylase levels, and (3) gallstones and/or sludge or a dilated common bile duct on imaging or elevated alanine aminotransferase (ALT) levels. The studied risk factors are shown in Table 1.

\section{Statistical Analysis}

Univariate logistic regression analysis was used to evaluate associations between potential risk factors and the occurrence of cholecystectomy. Additionally, all variables were entered in a multivariable logistic regression analysis (full model). Using stepwise backward elimination, a final model was derived (in each subsequent step, the least significant variable in the model was removed until all remaining variables have individual $p$ values smaller than 0.125 ).

The same procedure was performed in two subsets of the study population. The first subset consisted of cases who developed biliary symptoms within 9 months and controls since

Table 1 Association between patient characteristics and cholecystectomy

\begin{tabular}{|c|c|c|c|c|}
\hline & Cases $(n=233)$ & Controls $(n=466)$ & OR $(95 \% \mathrm{CI})$ & $p$ value \\
\hline Age in years, mean $\pm \mathrm{SD}$ & $39.8 \pm 10.6$ & $43.8 \pm 10.5$ & $0.97(0.95-0.98)$ & $<0.001$ \\
\hline Female, $n(\%)$ & $214(91.8)$ & $376(80.7)$ & $2.70(1.60-4.55)$ & $<0.001$ \\
\hline Fertile $^{1}, n(\%)$ & $183(85.5)$ & $285(75.8)$ & $1.89(1.20-2.95)$ & 0.006 \\
\hline Having children ${ }^{1}, n(\%)$ & $164(76.6)$ & $285(75.8)$ & $1.05(0.71-1.55)$ & 0.819 \\
\hline Caucasian, $n(\%)$ & $207(88.8)$ & $384(82.4)$ & $1.70(1.06-2.73)$ & 0.028 \\
\hline Physical active preoperative, $n(\%)$ & $55(23.6)$ & $111(23.8)$ & $0.99(0.68-1.43)$ & 0.950 \\
\hline Preoperative BMI, mean $\pm \mathrm{SD}\left(\mathrm{kg} / \mathrm{m}^{2}\right)$ & $43.4 \pm 5.1$ & $43.4 \pm 5.0$ & $1.00(0.97-1.03)$ & 0.839 \\
\hline$\% \mathrm{TWL}$ at 12 months, mean $\pm \mathrm{SD}$ & $35.1 \pm 6.7$ & $31.4 \pm 6.9$ & $1.08(1.06-1.11)$ & $<0.001$ \\
\hline Hypertension preoperative, $n(\%)$ & $112(48.1)$ & $284(60.9)$ & $0.59(0.43-0.82)$ & 0.001 \\
\hline Type 2 diabetes preoperative, $n(\%)$ & $31(13.3)$ & $108(23.2)$ & $0.51(0.33-0.79)$ & 0.002 \\
\hline Pain syndrome preoperative, $n(\%)$ & 27 (11.6) & $18(3.9)$ & $3.26(1.76-6.06)$ & $<0.001$ \\
\hline Preoperative use of statins, $n(\%)$ & $18(7.7)$ & $90(19.3)$ & $0.35(0.21-0.60)$ & $<0.001$ \\
\hline Preoperative use of statins, $n(\%)$ & & & & $0.003^{*}$ \\
\hline No & $215(92.3)$ & $376(80.7)$ & Reference & \\
\hline Low intensity & $8(3.4)$ & $24(5.2)$ & $0.58(0.26-1.32)$ & 0.196 \\
\hline Moderate intensity & $9(3.9)$ & $41(8.8)$ & $0.38(0.18-0.81)$ & 0.011 \\
\hline High intensity & $1(0.4)$ & $25(5.4)$ & $0.07(0.01-0.52)$ & 0.009 \\
\hline Preoperative use of birth control pills, $n(\%)$ & $62(29.0)$ & $75(19.9)$ & $1.64(1.11-2.42)$ & 0.013 \\
\hline
\end{tabular}

$O R$, odds ratio; $C I$, confidence interval; $S D$, standard deviation; $B M I$, body mass index; $T W L$, total weight loss

${ }^{1}$ Female patients only $(n=590)$

*We also performed a test for trend: OR $(95 \%$ CI) $0.55(0.41-0.73), p$ value $<0.001$ 
Fig. 1 Kaplan-Meier curve showing time to onset of biliary symptoms

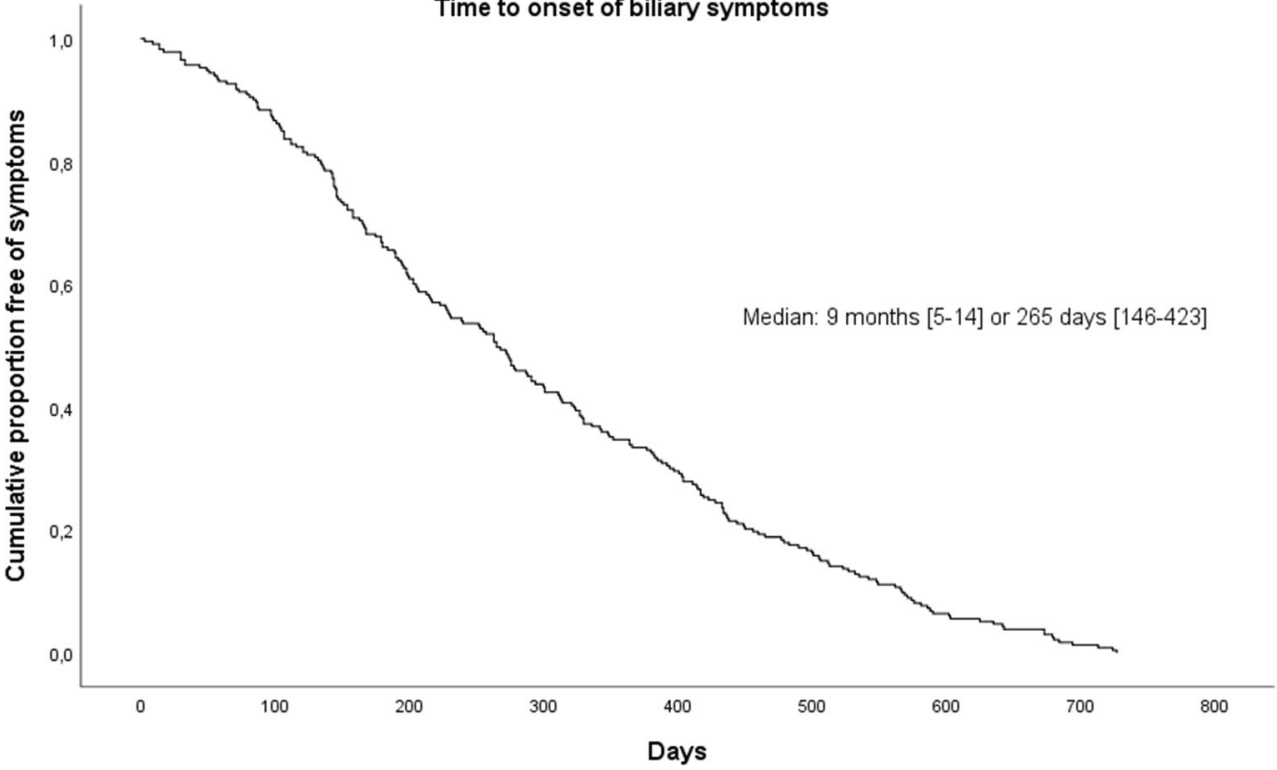

we hypothesized that we would observe a stronger association between potential risk factors and the occurrence of cholecystectomy in patients who developed biliary symptoms shortly after bariatric surgery. The second subset of patients consisted of female cases and controls in which we studied femaleassociated factors (fertility, offspring, use of birth control pills).

To test for a dose-effect relationship between the intensity of preoperative statin and cholecystectomy, the categorical variable "preoperative use of statins (no, low, moderate, high)" was entered as a continuous variable in our logistic regression models.

Kaplan-Meier curves were constructed to explore the "time to onset of biliary symptoms" and "time to cholecystectomy," separately. Time to onset of biliary symptoms or cholecystectomy was defined as the period from the date of bariatric surgery to the onset of biliary symptoms or date of cholecystectomy.

In the uni- and multivariable analyses we replaced/recoded missing values by using the mean of the values of the case or control group for numerical variables and "no" for categorical variables. In case the specific dates of the development of biliary symptoms or cholecystectomy were missing, they were estimated based on the visits of the outpatient clinic.

All statistical tests were two-sided and a $p$ value of less than 0.05 was considered significant. Statistical analyses were performed using IBM SPSS version 24 (SPSS Inc., Chicago, Ill).

\section{Results}

\section{Study Population}

Between January 2013 and December 2015, 1780 primary LRYGBs were performed at the MC Slotervaart. We identified $233(13.1 \%)$ cases who underwent a cholecystectomy because of biliary symptoms within 2 years after LRYGB. These cases were compared with 466 controls. Demographic and clinical characteristics of the study population are summarized in Table 1 . The mean age ( \pm standard deviation (SD)) of cases was $39.8 \pm 10.6$ and the mean age of controls was $43.8 \pm 10.5$ years. In both groups, most patients were female $(91.8 \%$ versus $80.7 \%)$ and the majority were Caucasian (88.8\% versus $82.4 \%)$. Preoperative BMI $( \pm$ SD) was similar $\left(43.4 \pm 5.1\right.$ and $\left.43.4 \pm 5.0 \mathrm{~kg} / \mathrm{m}^{2}\right)$.

During the first 24 postoperative months, cases developed biliary symptoms after a median (IQR) follow-up of 9 (5-14) months, and cholecystectomy was performed after a median follow-up of 12 (8-17) months (Figs. 1 and 2). The most common indication for cholecystectomy was biliary colic $(67.4 \%)$. Other indications were suspicion on biliary colic according to the treating surgeon, but not fitting the Rome criteria $(10.3 \%)$, cholecystitis $(7.7 \%)$, biliary pancreatitis $(1.7 \%)$, choledocholithiasis $(0.9 \%)$, and for $12 \%$ of the patients the indication was not specified.

\section{Risk Factors for Cholecystectomy}

The results of the univariate logistic regression analysis of all patients are shown in Table 1. Female gender (odds ratio (OR) $95 \%$ confidence interval $(95 \%$ CI) 2.70 (1.60-4.55)), Caucasian ethnicity (OR (95\% CI) 1.70 (1.06-2.73)), higher $\%$ TWL (OR (95\% CI) 1.08 (1.06-1.11)), and the existence of a preoperative pain syndrome (OR (95\%CI) $3.26(1.76-6.06))$ were significantly associated with an increased risk for subsequent cholecystectomy. Older age (OR (95\% CI) 0.97 (0.950.98)), hypertension (OR (95\% CI) 0.59 (0.43-0.82)), T2D (OR (95\% CI) $0.51(0.33-0.79))$, and the use of statins 
Fig. 2 Kaplan-Meier curve showing time to cholecystectomy

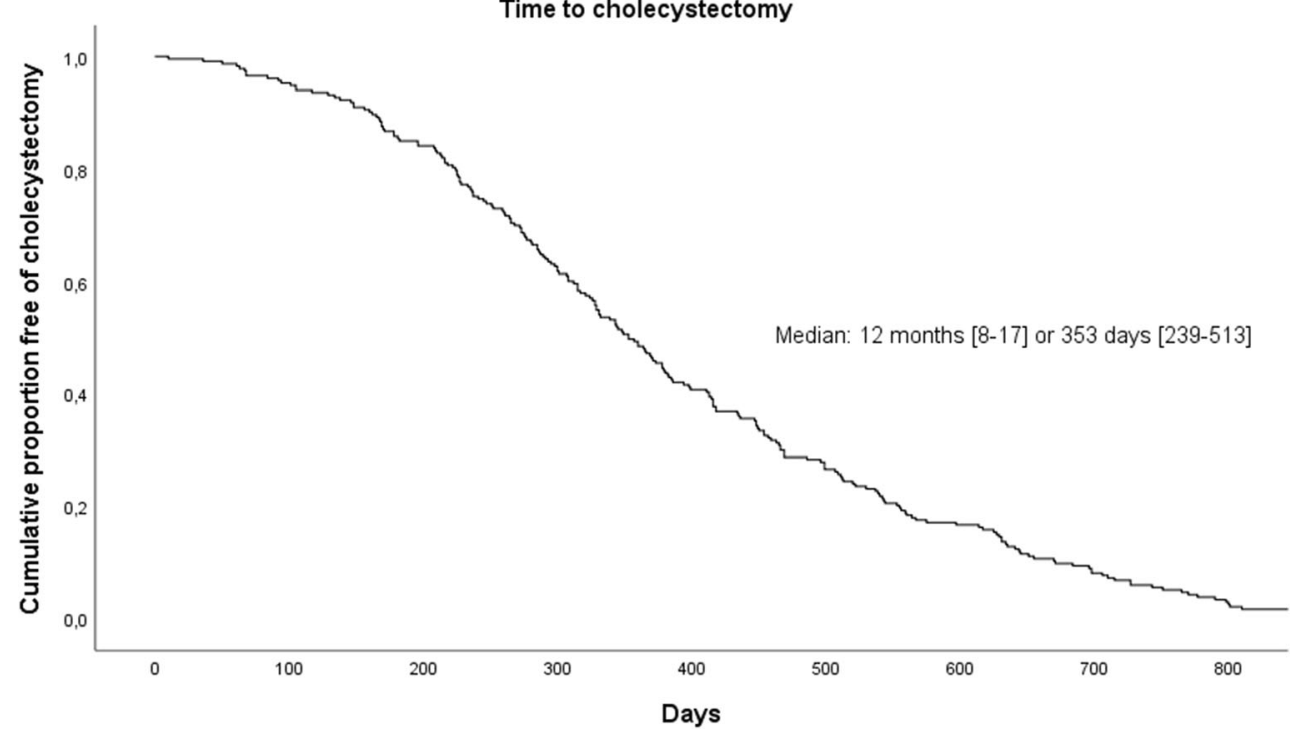

preoperatively (OR $(95 \% \mathrm{CI}) 0.35(0.21-0.60))$ were significantly associated with a reduced risk. When further exploring the intensity of statins, we observed a doseeffect relationship (overall $p$ value $=0.003$ ). As compared to no preoperative statin use, the use of low-intensity statin was not statistically significantly associated (OR $(95 \%$ CI) $0.58(0.26-1.32))$, while moderate-intensity statin (OR $(95 \%$ CI) $0.38(0.18-0.81))$ or high-intensity statin (OR $(95 \% \mathrm{CI}) 0.07(0.01-0.52))$ was associated with cholecystectomy. Testing for a trend confirmed the doseeffect relationship $(p<0.001)$. Preoperative physical activity and BMI were not associated with the risk for cholecystectomy.

The results of the multivariable logistic regression analysis in all patients are shown in Table 2. In the final model, four factors were associated with cholecystectomy after LRYGB: female gender (OR (95\% CI) 1.83 (1.06-3.17)), Caucasian ethnicity (OR (95\%) $1.82(1.10-3.02)$ ), \% TWL at 12 months (OR (95\%CI) 1.06 (1.04-1.09)), and pain syndrome (OR (95\% CI) $2.72(1.43-5.18)$ ). Older age (OR (95\%CI) 0.98 (0.96-0.99)) was found to be a protective factor, while preoperative statin use (OR (95\% CI) $0.56(0.31-1.00))$ almost reached statistical significance.

The dose-effect relationship of preoperative statin use and cholecystectomy was also established in the multivariable model, although not significant (overall $p$ value $=0.125$ ) . (Supplementary Table 1). Testing for a trend in this model confirmed the dose-effect $(p=0.013)$.

Table 3 summarizes the results of the multivariable analysis of cases who developed biliary symptoms within 9 months (median) after LRYGB. In this subgroup, preoperative use of statins was significantly associated (OR (95\% CI) 0.27
Table 2 Multivariable association between patient characteristics and cholecystectomy; all patients $(n=$ 699)

\begin{tabular}{|c|c|c|c|c|}
\hline & \multicolumn{2}{|l|}{ Full model } & \multicolumn{2}{|l|}{ Final model } \\
\hline & OR $(95 \% \mathrm{CI})$ & $p$ value & OR $(95 \% \mathrm{CI})$ & $p$ value \\
\hline Age in years, mean $\pm \mathrm{SD}$ & $0.98(0.96-0.99)$ & 0.011 & $0.98(0.96-0.99)$ & 0.005 \\
\hline Female, $n(\%)$ & $1.80(1.03-3.13)$ & 0.038 & $1.83(1.06-3.17)$ & 0.031 \\
\hline Caucasian, $n(\%)$ & $1.81(1.09-3.01)$ & 0.021 & $1.82(1.10-3.02)$ & 0.019 \\
\hline Physical active preoperative, $n(\%)$ & $1.00(0.67-1.49)$ & 0.999 & - & - \\
\hline Preoperative BMI, mean $\pm \mathrm{SD}\left(\mathrm{kg} / \mathrm{m}^{2}\right)$ & $0.99(0.95-1.02)$ & 0.449 & - & - \\
\hline$\% \mathrm{TWL}$ at 12 months, mean $\pm \mathrm{SD}$ & $1.06(1.04-1.09)$ & $<0.001$ & $1.06(1.04-1.09)$ & $<0.001$ \\
\hline Hypertension preoperative, $n(\%)$ & $0.91(0.63-1.83)$ & 0.592 & - & - \\
\hline Type 2 diabetes preoperative, $n(\%)$ & $1.08(0.64-1.83)$ & 0.771 & - & - \\
\hline Pain syndrome preoperative, $n(\%)$ & $2.63(1.38-5.03)$ & 0.003 & $2.72(1.43-5.18)$ & 0.002 \\
\hline Preoperative use of statins, $n(\%)$ & $0.55(0.29-1.04)$ & 0.067 & $0.56(0.31-1.00)$ & 0.051 \\
\hline
\end{tabular}

$O R$, odds ratio; $C I$, confidence interval; $S D$, standard deviation; $B M I$, body mass index; $T W L$, total weight loss 
Table 3 Multivariable association between patient characteristics and cholecystectomy; cases with biliary symptoms within 9 months and all controls $(n=$ 583)

\begin{tabular}{|c|c|c|c|c|}
\hline & \multicolumn{2}{|l|}{ Full model } & \multicolumn{2}{|l|}{ Final model } \\
\hline & OR $(95 \% \mathrm{CI})$ & $p$ value & OR $(95 \% \mathrm{CI})$ & $p$ value \\
\hline Age in years, mean $\pm S D$ & $0.97(0.94-0.99)$ & 0.003 & $0.97(0.95-0.99)$ & 0.003 \\
\hline Female, $n(\%)$ & $2.15(0.97-4.75)$ & 0.060 & $2.16(0.98-4.73)$ & 0.055 \\
\hline Caucasian, $n(\%)$ & $1.47(0.79-2.72)$ & 0.221 & - & - \\
\hline Physical active preoperative, $n(\%)$ & $0.94(0.56-1.58)$ & 0.811 & - & - \\
\hline Preoperative BMI, mean $\pm \mathrm{SD}\left(\mathrm{kg} / \mathrm{m}^{2}\right)$ & $0.97(0.92-1.01)$ & 0.165 & - & - \\
\hline$\% \mathrm{TWL}$ at 12 months, mean $\pm \mathrm{SD}$ & $1.07(1.04-1.11)$ & $<0.001$ & $1.07(1.04-1.11)$ & $<0.001$ \\
\hline Hypertension preoperative, $n(\%)$ & $0.91(0.57-1.45)$ & 0.687 & - & - \\
\hline Type 2 diabetes preoperative, $n(\%)$ & $1.15(0.56-2.36)$ & 0.707 & - & - \\
\hline Pain syndrome preoperative, $n(\%)$ & $2.61(1.19-5.73)$ & 0.017 & $2.70(1.23-5.92)$ & 0.013 \\
\hline Preoperative use of statins, $n(\%)$ & $0.25(0.08-0.78)$ & 0.017 & $0.27(0.09-0.78)$ & 0.016 \\
\hline
\end{tabular}

$O R$, odds ratio; $C I$, confidence interval; $S D$, standard deviation; $B M I$, body mass index; $T W L$, total weight loss
$(0.09-0.78))$, besides age, gender, \% TWL, and the existence of pain syndrome.

In the female population, female-associated factors (fertility, offspring, and the use of birth control pills) were not associated with cholecystectomy in the multivariable analysis (Supplementary Table 2). On the other hand, preoperative use of statins was associated, along with age, Caucasian ethnicity, \% TWL, and pain syndrome.

\section{Discussion}

In our hospital, $13.1 \%$ of patients developed postoperative biliary symptoms within 24 months after LRYGB and underwent subsequently a cholecystectomy. This percentage is consistent with the current literature. Several previous studies investigated risk factors for ultrasound-confirmed gallstones. Our study focused on cholecystectomy because this is more relevant for clinical practice. We found four independent risk factors for cholecystectomy after bariatric surgery: female gender, Caucasian ethnicity, \%TWL at 12 months, and preoperative pain syndrome.

Female gender is a well-known risk factor in general population $[1,12]$. Our study showed that also after bariatric surgery, women are at increased risk to develop biliary symptoms as compared with men. This is in accordance with the prospective study of Shiffman et al. who demonstrated that female gender is a risk factor for ultrasound-confirmed gallstones in bariatric patients [3]. However, the majority of patients undergoing bariatric surgery are female (in our cohort $84 \%$ ).

In line with Tsirline et al., we found that Caucasian ethnicity is a risk factor for cholecystectomy after bariatric surgery [14]. Historically, the prevalence of gallstones in Caucasians is higher than in African or Asian populations. The increased risk is probably due to genetic predisposition besides contributing environmental factors [1].
Not surprisingly, we found that \% TWL is positively associated with cholecystectomy. This means that the risk is substantially lower in patients with limited weight loss (\% TWL of $15 \%$ ) and substantially higher in patients with more than expected weight loss (\%TWL of $45 \%$ or more), compared with patients with average weight loss (\%TWL of 33\%). The observed odds ratio indicates that patients with limited weight loss will have a more than 2-fold lower risk for symptomatic gallstones and patients with more than expected weight loss a 2-fold higher risk, compared with patients with average weight loss. Percent TWL is independent of BMI and therefore a robust measure for weight change achieved per patient owing to bariatric surgery [23]. We studied \% TWL at 12 months (window 6-18 months) because most weight loss occurs within the first year postoperatively. In our study, no association was found for preoperative BMI, which is consistent with previous studies [4, 13, 14, 17]. Rapid weight loss probably induces changes in cholesterol metabolism. After bariatric surgery, hepatic cholesterol hypersecretion and/or hypo secretion of bile acids, or phospholipids causes an excess of biliary cholesterol. Also gallbladder hypomobility, an increase in pro-nucleating factors, and an altered intestinal mobility play a contributing role in the formation of gallstones [10, 11].

Finally, we found that the existence of a pain syndrome before surgery increases the chance of undergoing cholecystectomy because of abdominal complaints allocated to the presence of gallstones. To our knowledge, we are the first group who investigated this variable, although this result should be interpreted with caution because of the small number of patients who suffered from preoperative pain syndrome. It is generally known that nonspecific abdominal complaints are very common after bariatric surgery. We assume that patients with pain syndrome are more likely to search for help, often resulting in imaging of the abdomen. The probability to find gallstones during imaging is high, as $30-45 \%$ of patients will develop asymptomatic gallstones after bariatric surgery 
$[2,3]$. When a patient and a surgeon are aware of the presence of gallstones, abdominal symptoms might be more easily labeled as biliary colic resulting in cholecystectomy.

Interestingly, we found older age to be a significant protective factor instead of risk factor, while older age is a major risk factor in general population $[1,12]$. This might be explained by the fact that we excluded all patients who had undergone prior cholecystectomy. In our institute, around $15-20 \%$ of patients already have undergone cholecystectomy prior to bariatric surgery. Thus, there is a possibility that persons prone for developing gallstones with an advanced age already developed symptomatic gallstone disease earlier in life and were not included in our study population.

Preoperative use of statins seemed to be a protective factor for cholecystectomy. In theory, statins may reduce the risk of gallstones by diminishing hepatic cholesterol levels but in literature the effect has not been consistently demonstrated $[24,25]$. Recently, findings from a large sample of the Danish population were presented, which showed that genetic variation in $\mathrm{HMGco} A$ reductase gene leading to lower LDL-C levels as well as statin use were both associated with a lower risk of gallstone disease, suggestive of causality [26]. We are the first study who investigated the effect of statins in patients after bariatric surgery. In the entire study population, preoperative use of statins almost reached statistical significance ( $p=$ 0.051 ), while the association was significant in two subsets of the study population. We also found a dose-effect relationship. Unfortunately, we lacked statistical power because only a small number of patients used statins, especially in the group of cases which might not be due to chance. If the association between use of higher dose of statins and gallstone formation will be confirmed, the effect of prescribing moderate or highdose statins on gallstone formation, biliary symptoms, and cholecystectomy should be investigated.

Last but not least, we found no significant associations between three female-associated factors (fertility, offspring, and use of birth control pills) and cholecystectomy. This was consistent with a large cohort study conducted in a general population of Denmark [27].

One of the strengths of our study is the large number of patients who underwent a cholecystectomy because of biliary symptoms $(n=233)$, which is high compared with previous conducted studies. Most factors in our study were preoperatively measured and objective of nature resulting in a low risk of (non)differential misclassification. Furthermore, there was a low number of missing data. We only had to deal with 54 missing values $(7.7 \%)$ of the variable "physical activity" and 2 missing values $(0.3 \%)$ of \% TWL. These values were replaced by using the mean of the values of the case or control group for numerical variables and "no" for categorical variables. We have performed additional analyses in which patients with missing values were excluded. These analyses resulted in similar findings.
A limitation is the retrospective nature of this study. To reduce the risk of selection bias in our case control study, we selected controls consecutively based on date of operation of the case. Additionally, we may have missed some cases, for example patients who underwent cholecystectomy in another hospital and stopped visiting our outpatient clinic.

In conclusion, patients who are female, Caucasian, or patients with a preoperative pain syndrome are at risk for cholecystectomy after bariatric surgery. Therefore, these patients might benefit from preventative measures. However, whether ursodeoxycholic acid or statins are able to prevent cholecystectomy in these patients needs to be confirmed in prospective studies. As expected, \% TWL was also found to be a risk factor but unfortunately, it is still not possible to predict accurately how much weight a patient will lose after bariatric surgery.

Acknowledgments We acknowledge Arafi Amarti, an ICT employee at MC Slotervaart.

\section{Compliance with Ethical Standards}

Conflict of Interest The authors declare that they have no conflicts of interest.

Ethical Statement All procedures performed in this study involving human participants were in accordance with the ethical standards of the institutional and/or national research committee and with the 1964 Helsinki declaration and its later amendments or comparable ethical standards.

Consent Statement For this type of study (retrospective) formal consent is not required. However, all patients signed an informed consent form before undergoing bariatric surgery in our institution, specifically stating that their anonymized data can be used for scientific research.

Open Access This article is distributed under the terms of the Creative Commons Attribution 4.0 International License (http:// creativecommons.org/licenses/by/4.0/), which permits unrestricted use, distribution, and reproduction in any medium, provided you give appropriate credit to the original author(s) and the source, provide a link to the Creative Commons license, and indicate if changes were made.

\section{References}

1. Lammert F, Gurusamy K, Ko CW, et al. Gallstones. Nat Rev Dis Primers. 2016;2:16024.

2. Manatsathit W, Leelasinjaroen $\mathrm{P}, \mathrm{Al}-\mathrm{Hamid} \mathrm{H}$, et al. The incidence of cholelithiasis after sleeve gastrectomy and its association with weight loss: a two-centre retrospective cohort study. Int J Surg. 2016;30:13-8.

3. Shiffman ML, Sugerman HJ, Kellum JM, et al. Gallstone formation after rapid weight loss: a prospective study in patients undergoing gastric bypass surgery for treatment of morbid obesity. Am J Gastroenterol. 1991;86(8):1000-5.

4. Li VK, Pulido N, Fajnwaks P, et al. Predictors of gallstone formation after bariatric surgery: a multivariate analysis of risk factors 
comparing gastric bypass, gastric banding, and sleeve gastrectomy. Surg Endosc. 2009;23(7):1640-4.

5. Nagem R, Lazaro-da-Silva A. Cholecystolithiasis after gastric bypass: a clinical, biochemical, and ultrasonographic 3-year follow-up study. Obes Surg. 2012;22(10):1594-9.

6. Portenier DD, Grant JP, Blackwood HS, et al. Expectant management of the asymptomatic gallbladder at Roux-en-Y gastric bypass. Surg Obes Relat Dis. 2007;3(4):476-9.

7. Ackroyd R. Population-based study of the need for cholecystectomy after obesity surgery (Br J Surg 2012; 99: 864-869). Br J Surg. 2012;99(6):870.

8. Wanjura V, Sandblom G, Osterberg J, et al. Cholecystectomy after gastric bypass-incidence and complications. Surg Obes Relat Dis. 2017;13(6):979-87.

9. Choi EK, Chiorean MV, Cote GA, et al. ERCP via gastrostomy vs. double balloon enteroscopy in patients with prior bariatric Rouxen-Y gastric bypass surgery. Surg Endosc. 2013;27(8):2894-9.

10. Paumgartner G, Sauerbruch T. Gallstones: pathogenesis. Lancet. 1991;338(8775):1117-21.

11. Shiffman ML, Sugerman HJ, Kellum JM, et al. Changes in gallbladder bile composition following gallstone formation and weight reduction. Gastroenterology. 1992;103(1):214-21.

12. Portincasa P, Moschetta A, Palasciano G. Cholesterol gallstone disease. Lancet. 2006;368(9531):230-9.

13. D'Hondt M, Sergeant G, Deylgat B, et al. Prophylactic cholecystectomy, a mandatory step in morbidly obese patients undergoing laparoscopic Roux-en-Y gastric bypass. J Gastrointest Surg. 2011;15(9):1532-6.

14. Tsirline VB, Keilani ZM, El Djouzi S, et al. How frequently and when do patients undergo cholecystectomy after bariatric surgery? Surg Obes Relat Dis. 2014;10(2):313-21.

15. Coupaye M, Castel B, Sami O, et al. Comparison of the incidence of cholelithiasis after sleeve gastrectomy and Roux-en-Y gastric bypass in obese patients: a prospective study. Surg Obes Relat Dis. 2015;11(4):779-84.

16. Iglezias Brandao de Oliveira C, Adami Chaim E, da Silva BB. Impact of rapid weight reduction on risk of cholelithiasis after bariatric surgery. Obes Surg. 2003;13(4):625-8.
17. Chang J, Corcelles R, Boules M, et al. Predictive factors of biliary complications after bariatric surgery. Surg Obes Relat Dis. 2016;12(9):1706-10.

18. Beweegrichtlijnen 2017: Gezondheidsraad; 2017. Available from: Accessed 9 July 2018.

19. Law MR, Wald NJ, Rudnicka AR. Quantifying effect of statins on low density lipoprotein cholesterol, ischaemic heart disease, and stroke: systematic review and meta-analysis. BMJ. 2003;326(7404):1423-0.

20. Huijgen R, Kindt I, Verhoeven SB, et al. Two years after molecular diagnosis of familial hypercholesterolemia: majority on cholesterollowering treatment but a minority reaches treatment goal. PLoS One. 2010;5(2):e9220.

21. Yokoe M, Hata J, Takada T, et al. Tokyo guidelines 2018: diagnostic criteria and severity grading of acute cholecystitis (with videos). J Hepatobiliary Pancreat Sci. 2018;25(1):41-54.

22. Kiriyama S, Takada T, Strasberg SM, et al. TG13 guidelines for diagnosis and severity grading of acute cholangitis (with videos). $\mathrm{J}$ Hepatobiliary Pancreat Sci. 2013;20(1):24-34.

23. Nelson DW, Blair KS, Martin MJ. Analysis of obesity-related outcomes and bariatric failure rates with the duodenal switch vs gastric bypass for morbid obesity. Arch Surg. 2012;147(9):847-54.

24. Bodmer M, Brauchli YB, Krahenbuhl S, et al. Statin use and risk of gallstone disease followed by cholecystectomy. Jama. 2009;302(18):2001-7.

25. Erichsen R, Froslev T, Lash TL, et al. Long-term statin use and the risk of gallstone disease: a population-based case-control study. Am J Epidemiol. 2011;173(2):162-70.

26. Qayyum F, Lauridsen BK, Frikke-Schmidt R, et al. Statin treatment, genetic inhibition of HMGCR and risk of symptomatic gallstone disease. Atherosclerosis. 2018;275:e35.

27. Shabanzadeh DM, Sorensen LT, Jorgensen T. A prediction rule for risk stratification of incidentally discovered gallstones: results from a large cohort study. Gastroenterology. 2016;150(1):156-167 e151.

Publisher's Note Springer Nature remains neutral with regard to jurisdictional claims in published maps and institutional affiliations. 